\title{
Ultrafast Strong-Field Photoemission from Plasmonic Nanoparticles
}

\author{
Péter Dombi, ${ }^{* \dagger}$ Anton Hörl, ${ }^{\dagger}$ Péter Rácz, ${ }^{\dagger}$ István Márton, ${ }^{\dagger}$ Andreas Trügler, ${ }^{\ddagger}$ Joachim R. Krenn, ${ }^{\ddagger}$ \\ and Ulrich Hohenester* ${ }^{*}+$
}

\author{
${ }^{\dagger}$ Wigner Research Centre for Physics, Konkoly-Thege M. út 29-33, 1121 Budapest, Hungary \\ ${ }^{\ddagger}$ Institut für Physik, Karl-Franzens Universität Graz, Universitätsplatz 5, 8010 Graz, Austria
}

\section{Supporting Information}

\begin{abstract}
We demonstrate the ultrafast generation of electrons from tailored metallic nanoparticles and unravel the role of plasmonic field enhancement in this process by comparing resonant and off-resonant particles, as well as different particle geometries. We find that electrons become strongly accelerated within the evanescent fields of the plasmonic nanoparticles and escape along straight trajectories with orientations governed by the particle geometry. These results establish plasmonic nanoparticles as versatile ultrafast, nanoscopic sources of electrons.
\end{abstract}

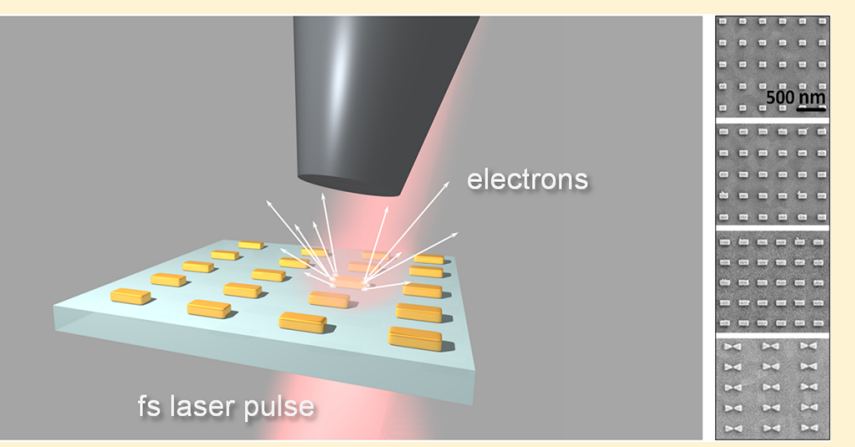

KEYWORDS: Plasmonics, nanoparticles, strong-field photoemission, lightwave electronics

$\mathrm{M}$ etallic nanoparticles sustain surface charge oscillations, so-called surface plasmons, which exhibit a pronounced resonance behavior and come together with strongly localized, evanescent fields. ${ }^{1,2}$ The combination of resonance and subwavelength field localization ${ }^{3}$ renders these particles ideal for various applications, including (bio)sensors, ${ }^{4}$ optoelectronics, ${ }^{5,6}$ photovoltaics, ${ }^{7}$ as well as nanotip-based ultrafast electron emitters, ${ }^{8-10}$ where upon illumination with femtosecond laser pulses electrons become photoemitted and ponderomotively accelerated. Even though the synergy between plasmonics and strong-field physics is still an exploratory area of fundamental research, recent achievements highlight the potential offered by nanolocalized, intense, and ultrashort electromagnetic wavepackets. ${ }^{11,12}$ These include alloptical acceleration of electrons up to $\mathrm{keV}$ levels in highly evanescent fields at metal films ${ }^{13,14}$ and clusters, ${ }^{15,16}$ the absorption of excess photons in the photoemission process at metallic nanotips ${ }^{17}$ (so-called above threshold photoemission), attosecond control of nanotip-emitted electrons via waveform shaping of the laser pulse, ${ }^{8}$ the quenching of the quiver motion of electrons at mid-infrared wavelengths, ${ }^{9}$ and the generation of high-order harmonics in the enhanced fields of nanostructures. ${ }^{18}$ A related work has also addressed the controlled nearfield enhanced electron acceleration from dielectric nanospheres. ${ }^{19}$ As opposed to field enhancement at chemically etched nanotips or with propagating surface plasmons (SPs) at metal films, we here show that plasmonic nanoparticles represent a versatile tool for controlling photoemission and photoacceleration on the nanoscale. Precisely fabricated nanoparticles offer the possibility of tailoring nanoscale electric fields with a high-level control over the plasmonic resonance, which can be overlapped with the spectrum of the exciting laser pulse to achieve field enhancements of the incident radiation by factors of up to several hundreds, ${ }^{3}$ thus offering an unparalleled possibility to investigate strong-field effects at low laser intensities with simple femtosecond oscillators. The effects of field enhancement and resonance behavior can be investigated by tuning the plasmonic resonance via the particle shape, without changing any other experimental parameter.

The concept of our experiment is schematically illustrated in Figure 1 and relies on the controlled fabrication of plasmonic gold nanoparticles on glass substrates, illuminated by linearly polarized femtosecond laser pulses of $95-110$ fs duration with a central wavelength of $805 \mathrm{~nm}$. After coupling the pulses into SP oscillations of the nanoparticles placed in vacuum, strongfield photoemission and photoacceleration processes are characterized by time-of-flight electron spectrometry (for more details see Supporting Information). A central element of the experimental concept is the fabrication of resonant and off-resonant nanorods with respect to the given laser wavelength, as well as of resonant bowtie nanostructures with significantly higher plasmonic field enhancement than for the nanorods. This way, both plasmonic resonance and field enhancement effects can be effectively correlated with photoemission spectra by switching between the different sample types.

We fabricated different sets of nanoparticle arrays with 0.01 $\mathrm{mm}^{2}$ surface area each, comprised of approximately 80000 noncoupled, rod-type nanoparticles on a conductive, transparent substrate. The nanorod height and width measured 40

Received: November 21, 2012

Revised: January 8, 2013

Published: January 22, 2013 

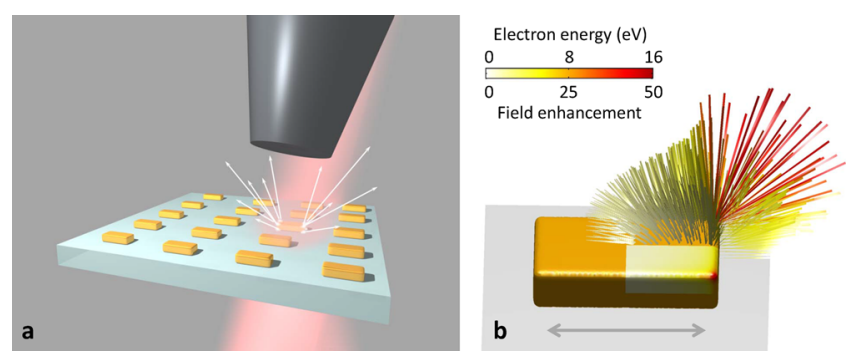

Figure 1. Scheme of photoemission from plasmonic nanoparticles. (a) A femtosecond laser pulse excites an array of virtually identical gold nanorods from below. The laser wavelength is matched with the plasmon resonance, and the polarization is aligned along the long axis of the nanorod (see arrow in panel b). In the strong plasmonic fields, electrons become photoemitted and ponderomotively accelerated and are finally analyzed by time-of-flight spectrometry. (b) Simulation results for semiclassical simple-man's model (same simulation parameters as in Figure 4). The lines pointing away from the nanorod report the electron trajectories; the colors indicate the final kinetic energies. The colored surface region (front right) on the rod shows the field enhancement for plane wave excitation of the laser.

and $87 \mathrm{~nm}$, respectively, and the length was $120 \mathrm{~nm}, 152 \mathrm{~nm}$, and $183 \mathrm{~nm}$ for three different arrays. Figure $2 \mathrm{~b}-\mathrm{d}$ shows scanning electron microscope (SEM) images of the arrays. Measured optical resonances of the different nanoparticle geometries are depicted in Figure 2a. Resonances are peaked between 732 and $877 \mathrm{~nm}$, and are blue-shifted, on-resonance and red-shifted, respectively, with respect to the laser bandwidth indicated by the dashed box.

Upon illumination with the femtosecond laser, electrons are generated in the nanoparticle fields, the spectra of which are shown in Figure $2 \mathrm{f}$ and Figure 3a-c. High-energy electrons with kinetic energies up to $19 \mathrm{eV}$ are observed, well above the photon energy of $1.54 \mathrm{eV}$ of the laser wavelength. It is also clear from Figure 3e that the cutoffs of the electron spectra scale approximately linearly with the intensity of the exciting pulses and that the highest photoemission yields and highest kinetic energies are observed from the plasmonically resonant nanoparticle array, followed by the red-shifted and blue-shifted nanorods, respectively. This already indicates the important role that the SP generation and corresponding field enhancement play in both the photoemission and the field-acceleration process. Due to the intricate relation ${ }^{20}$ between the far-field peak intensities of plasmonic nanoparticles and the corresponding near fields, which govern the ponderomotive electron acceleration, we here refrain from a direct comparison of the optical spectra and cutoff energies shown in Figures 2 and 3, respectively, but will discuss this point in more detail elsewhere.

These findings are further corroborated by additional experiments performed with resonant coupled nanotriangle pairs (bowtie geometry), which are known to exhibit a much stronger field enhancement in the gap region between the nanotriangles. ${ }^{3}$ The gaps of the bowtie geometry are $20 \mathrm{~nm}$ with variation throughout the array measured to be approximately $\pm 2 \mathrm{~nm}$. The results of the measurements, with the same experimental conditions as for the nanorods, together with the particle geometries and optical resonance properties are shown in Figures 2 and 3 (green curves). It is clearly visible that in this case significantly lower incident laser intensities are sufficient to generate the same photoelectron yield and electron energies as for the nanorods. Carrying out experiments with intensities above $25 \mathrm{GW} / \mathrm{cm}^{2}$ were rendered impossible by
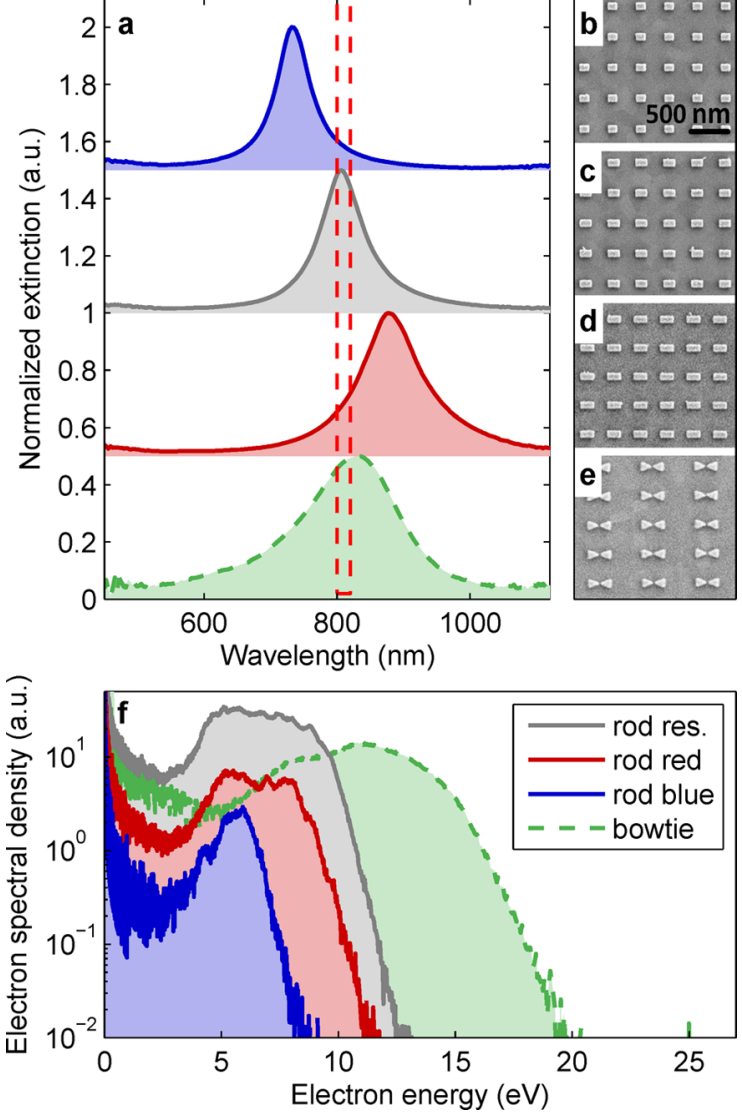

Figure 2. Measured optical spectra and electron kinetic energy distributions for nanorods and bowtie nanoparticles. (a) Measured extinction spectra for nanorods with dimensions of (b) $120 \times 87 \times 40$ $\mathrm{nm}^{3}$, (c) $152 \times 87 \times 40 \mathrm{~nm}^{3}$, and (d) $183 \times 87 \times 40 \mathrm{~nm}^{3}$, which are blue-shifted, in resonance, and red-shifted with respect to the excitation bandwidth centered at $\lambda_{\text {exc }}=805 \mathrm{~nm}$ (see dashed box). Spectra are offset for clarity. The bottom curve in panel a reports the spectrum for a bowtie structure with $90 \mathrm{~nm}$ width, $40 \mathrm{~nm}$ height, and $260 \mathrm{~nm}$ length (20 nm gap). (f) Electron spectra for different particle geometries and for a laser peak intensity of $25.1 \mathrm{GW} / \mathrm{cm}^{2}$. The data below $3 \mathrm{eV}$ are of limited validity due to instrumental restrictions of the time-of-flight spectrometer.

optical damage of the nanoparticles, also indicating higher electric field enhancement than in the case of nanorods.

To analyze the experimental results and correlate them quantitatively with plasmonic field enhancement, we performed simulations with the MNPBEM toolbox ${ }^{21}$ which is based on a boundary element method (BEM) approach. ${ }^{22}$ We start by computing the optical spectra for the metallic nanoparticles using a gold dielectric function extracted experimental ${ }^{23}$ and evaluated at the wavelength $\lambda_{\mathrm{exc}} \approx 800 \mathrm{~nm}$ of the exciting laser, finding good agreement between the simulated and measured extinction spectra (see Supporting Information). The ponderomotive acceleration of electrons is simulated within the semiclassical simple man's model. ${ }^{9,24,25}$ In a first step, electrons become photoemitted. We use an expression for nonadiabatic tunnel ionization, ${ }^{26}$ which interpolates between the perturbative regime of multiphoton emission ${ }^{14,27}$ for large Keldysh parameters ${ }^{28} \gamma$ and tunneling for $\gamma<1$. Since the electric field varies strongly along the surface of the nanoparticle, both photoemission mechanisms need to be taken into account. In a second step, the photoemitted electrons are accelerated by the total field of the external laser pulse together with the induced 

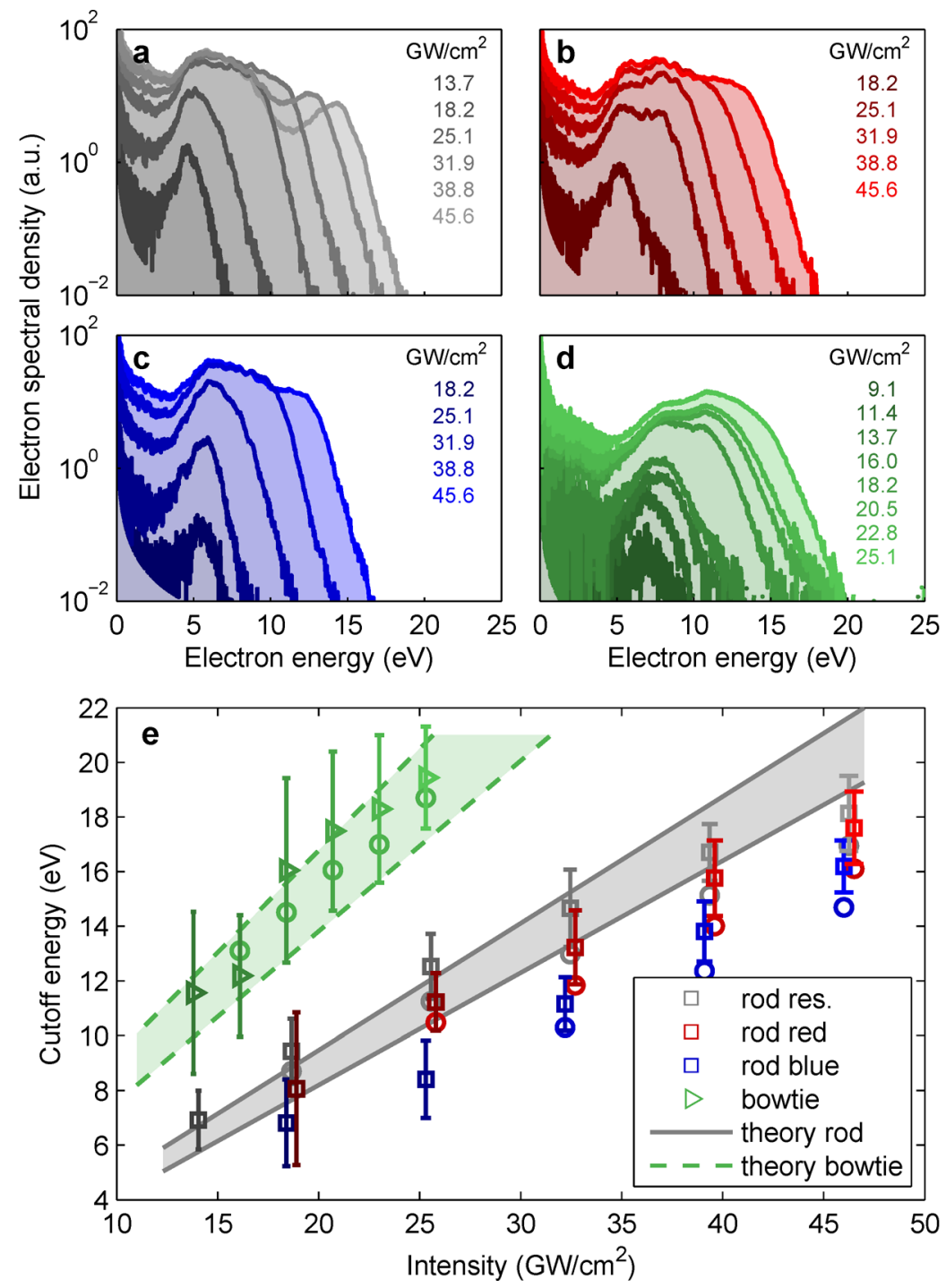

Figure 3. Electron spectra as a function of peak intensity for (a) resonant rod, (b) red-shifted rod, (c) blue-shifted rod, and (d) resonant bowtie structure. (e) Cutoff energies $\varepsilon_{\text {cut }}$, where the electron distribution $f(\varepsilon)$ lies within 1 and $5 \%$ of its maximum value above $3 \mathrm{eV}$ (range of cutoff energies according to errorbar), as a function of laser intensity. The circles show for comparison also $\varepsilon_{\text {cut }}$ obtained from $f\left(\varepsilon_{\text {cut }}\right)=f\left(\varepsilon_{\text {cut }} / 2\right) \times 10^{-2}$, according to the prescription of ref 19 . The solid and dashed lines show simulation results for the resonant rod and bowtie nanoparticle, respectively. The influence of different cutoff angles $\theta_{\text {cut }}=6-10^{\circ}$ (accounting for the acceptance cone of the electron spectrometer) on the cutoff energies is indicated by the shaded areas.

(evanescent) field of the plasmonic nanoparticle. Contrary to previous studies, which assumed a homogeneous ${ }^{8}$ or a simplified dipolar-like field ${ }^{9}$ at nanotips, in our approach the full plasmonic fields are computed at each position of the electron trajectory.

Figure $1 \mathrm{~b}$ shows simulated electron trajectories, together with the plasmonic field enhancement, for a rod-like nanoparticle (dimensions $160 \times 80 \times 40 \mathrm{~nm}^{3}$ ) whose resonance wavelength matches $\lambda_{\text {exc }}$. The radii of curvature of the corners and edges are $\sim 5 \mathrm{~nm}$, which constitutes a representative value for nanoparticles produced by electron beam lithography. ${ }^{30}$ As can be seen in the figure, the fastest electrons emerge from the hot spots of the particle where the nearfield enhancement is maximal (here the corners of the rod). In addition, the electrons escape along almost straight lines. Figure $4 c$ shows details of the transient energies for electrons originating from the corners, edges, and flat surfaces of the nanorod. The main acceleration occurs within the first cycle of $E_{\text {ind }}$, where the extremely localized (evanescent) field acts strongest on the electrons (typical 1/e decay lengths of the optical fields at the hot spots are $l_{F} \approx 30 \mathrm{~nm}$, whereas electron quiver amplitude is around $\left.1 \mathrm{~nm}^{9}\right)$. As the evanescent fields approximately point along the directions normal to the surface (as expected for ideal conductors where $\boldsymbol{E}$ is normal to the surface), the directions of the electron trajectories are completely governed by the surface geometry at the hot spots. ${ }^{29}$ On resonance the induced field $\boldsymbol{E}_{\text {ind }}$ is delayed by $90^{\circ}$ with respect to the driving field $\boldsymbol{E}_{\mathrm{ext}}$, and the highest electron energies arise when the acceleration force in the direction away from the particle acts for a complete halfcycle. This applies to electrons photoexcited at the minimum as well as around the maximum of $\boldsymbol{E}_{\text {ind }}$. In the latter case, electrons are initially pushed away from the surface, until $\boldsymbol{E}_{\text {ind }}$ changes sign and the electrons become accelerated toward the surface, where they suffer reflection and finally undergo again acceleration (but now with a higher initial energy) in the consecutive half-cycle of $\boldsymbol{E}_{\text {ind }}$ (see Supporting Information). 

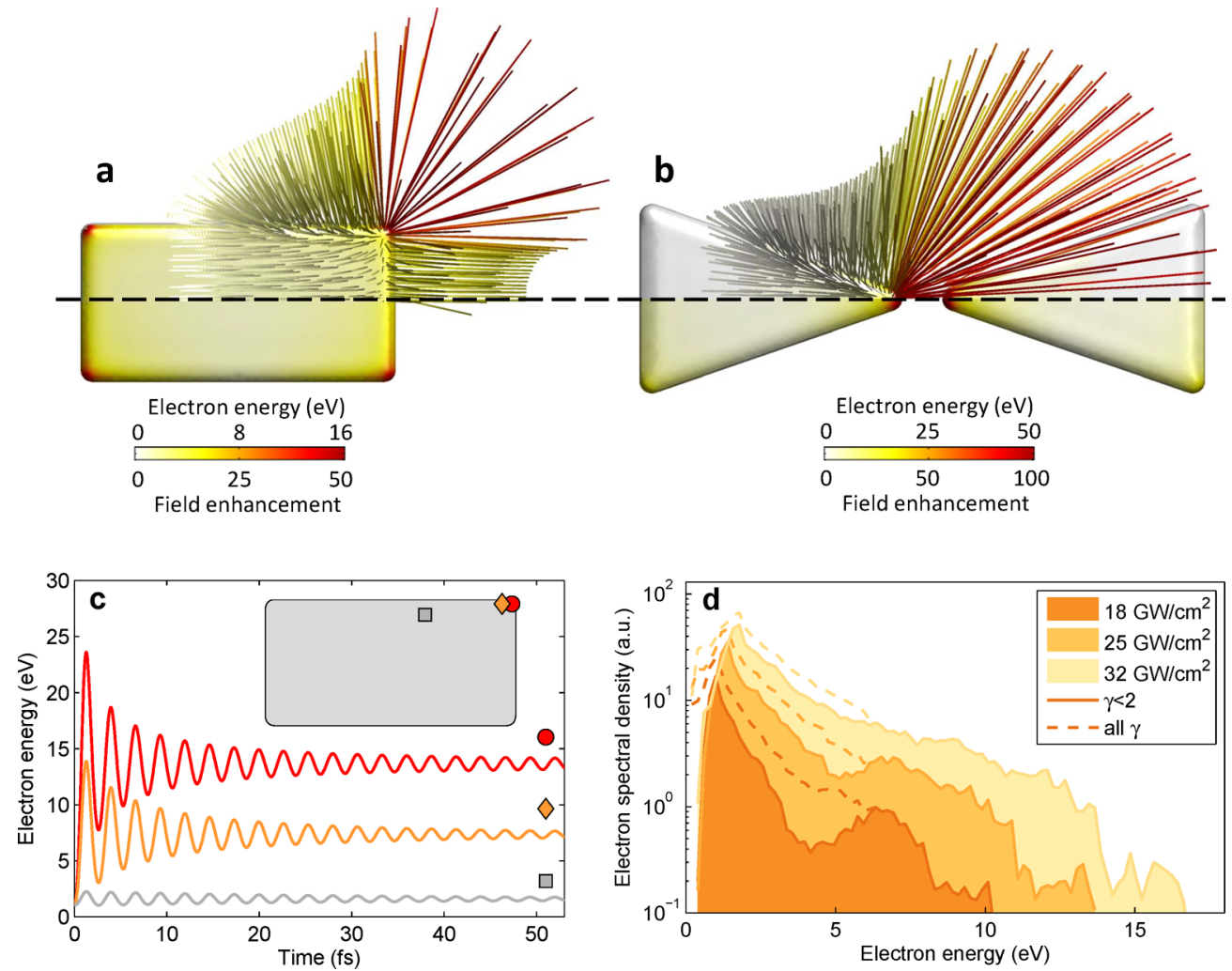

Figure 4. Simulation results. (a) The upper part (above dashed line) reports the electron trajectories, with colors chosen according to the final kinetic energies of the electrons. The color on the particle surface corresponds to the final kinetic energy of the electron photoemitted from the respective spot. The lower part shows the absolute value of the field enhancement $f=E_{\text {ind }} / E_{\text {ext }}$ on the nanoparticle surface. (b) Same as panel a but for bowtie particle. (c) Time dependence of electron energies for electrons originating from the spots indicated by the symbols in the inset. For all simulations the laser intensity is set to $35 \mathrm{GW} / \mathrm{cm}^{2}$, corresponding to a maximal field strength of $E_{\text {ext }} \approx 0.5 \mathrm{~V} / \mathrm{nm}$ (Keldysh parameter of 0.66 for field enhancement of 50), and the electrons are photoexcited at the maximum of $E_{\text {ext }}$ (d) Simulated electron distribution for nanorod and for different excitation powers (dashed lines). The solid lines show spectra for the electrons photoexcited at the largest field strengts (Keldysh parameter $\gamma<2$ ). In the simulations we assume a Gaussian envelope for the exciting laser pulse and use $\theta_{\text {cut }}=10^{\circ}$.

Such rescattering phenomena have been demonstrated to be of importance also in atomic systems as well as for metal tips excited in the absence of strong plasmonic field enhancement. $^{8,9,31}$

To compute the electron distributions, one has to weigh the contributions of the electrons by the photexcitation probabilities $P$ (see Supporting Information). Figure $4 \mathrm{~d}$ reports the simulated electron spectral densities and Figure $3 \mathrm{e}$ the extracted cutoff energies. The characteristic features of the distributions agree well with those of the experimental spectra. At low kinetic energies one sees a well-known direct peak, ${ }^{17}$ which is followed by a decaying part (with a second peak for the experimental spectra) attributed to the region where direct and rescattered electrons both contribute to the emission. ${ }^{31}$ This spectral region is particularly challenging to reproduce in the simulations, even with quantum mechanical models, due to various reasons including interferences between emission channels. $^{31}$ Finally, a cutoff is observed, signifying the maximum ponderomotive acceleration, the position of which is accounted for by our semiclassical simulations. Our model thus reproduces the classical features in the plasmonic acceleration process very well. Better agreement between the experimental and the simulation results can be achieved if only those photoemission events are considered where $\gamma<2$ holds (i.e., mainly photoexcitation at the hot spots). This suggests that the photoemission formula used ${ }^{26}$ underestimates the weight of tunneling emission events and has limitations for describing photoemission from metals, as also supported by a recent related analysis. ${ }^{32}$ This is corroborated by the fact that the nonadiabatic tunneling formula predicts a much slower transition between muti-photon-induced and tunneling emission (with respect to the intensity) than recent experimental evidence. ${ }^{14,33}$ Yet, it still gives better results than alternative static-field or other atomic tunneling expressions where the complexity of the field distribution around the nanoparticles and the simultaneous presence of tunneling and multiphoton emission events cannot be taken into account with a single closed-form expression.

In summary, we have demonstrated generation of electrons induced by ultrashort laser pulses in the vicinity of tailored plasmonic nanostructures. The photoacceleration process was shown to be governed by the evanescent surface plasmon field of the nanoparticle, which allows for a high-level control of electron emission by tailoring the geometry and thereby the plasmonic particle resonances. Plasmonic particles can be integrated in monolithic devices and can be combined with other nanophotonic components, thus offering unique prospects for the generation and all-optical control of plasmonic electron sources as well as other applications in lightwave electronics. Our experiments additionally open new possibilities for the investigation of femtosecond and attosecond processes in nanosystems and time-resolved studies of collective electron motion in solids. 


\section{ASSOCIATED CONTENT}

\section{S Supporting Information}

Further details about the experiments and simulations. This material is available free of charge via the Internet at http:// pubs.acs.org.

\section{AUTHOR INFORMATION}

\section{Corresponding Author}

*E-mail: dombi.peter@wigner.mta.hu; ulrich.hohenester@unigraz.at.

\section{Notes}

The authors declare no competing financial interest.

\section{ACKNOWLEDGMENTS}

This work was supported by the Austrian Science Fund FWF under projects No. P21235-N20 and P24511-N26. The authors wish to acknowledge Christine Prietl for sample fabrication and Júlia Fekete for participating in the calibration of the electron spectrometer. P.D. was supported by the János Bolyai Research Scholarship of the Hungarian Academy of Sciences.

\section{REFERENCES}

(1) Atwater, H. Sci. Am. 2007, 296 (4), 56-63.

(2) Maier, S. A. Phasmonics: Fundamentals and Applications; Springer, Berlin, 2007.

(3) Schuller, J. A.; Barnard, E. S.; Cai, W.; Jun, Y. C.; White, J. S.; Brongersma, M. L. Nat. Mater. 2010, 9, 193-204.

(4) Anker, J. N.; Hall, W. P.; Lyandres, O.; Shah, N. C.; Zhao, J.; Van Duyne, R. P. Nat. Mater. 2008, 7, 442-453.

(5) Koller, D.; Hohenau, A.; Ditlbacher, H.; Galler, N.; Reil, F.; Aussenegg, F. R.; Leitner, A.; List, E.; Krenn, J. R. Nat. Photonics 2008, 2, 684-687.

(6) Falk, A. L.; Koppens, F. H. L.; Yu, C. L.; Kang, K.; de Leon Snapp, N.; Akimov, A. V.; Jo, M.-H.; Lukin, M. D.; Park, H. Nat. Phys. 2009, 5, 475-479.

(7) Ferry, V. E.; Sweatlock, L. A.; Pacifci, D.; Atwater, H. A. Nano Lett. 2008, 8, 4391-4397.

(8) Krüger, M.; Schenk, M.; Hommelhoff, P. Nature 2011, 475, 7881.

(9) Herink, G.; Solli, D. R.; Gulde, M.; Ropers, C. Nature 2012, 483, 190-193.

(10) Park, D. J.; Piglosiewicz, B.; Schmidt, S.; Kollmann, H.; Mascheck, M.; Lienau, C. Phys. Rev. Lett. 2012, 109, 244803.

(11) Aeschlimann, M.; Bauer, M.; Bayer, D.; Brixner, T.; Garcia de Abajo, F. J.; Pfeiffer, W.; Rohmer, M.; Spindler, C.; Steeb, F. Nature 2007, 446, 301-304.

(12) Stockman, M.; Kling, M. F.; Krausz, F.; Kleineberg, U. Nat. Photonics 2007, 1, 539-544.

(13) Irvine, S. E.; Dechant, A.; Elezzabi, A. Y. Phys. Rev. Lett. 2004, 93, 184801.

(14) Dombi, P.; Irvine, S. E.; Racz, P.; Lenner, M.; Kroo, N.; Farkas, G.; Mitrofanov, A.; Baltuska, A.; Fuji, T.; Krausz, F.; Elezzabi, A. Y. Opt. Express 2010, 18, 24206-24212.

(15) Fennel, Th.; Döppner, T.; Passig, J.; Schaal, Ch.; Tiggesbäumker, J.; Meiwes-Broer, K.-H. Phys. Rev. Lett. 2007, 98, 143401.

(16) Word, R. C.; Fitzgerald, J.; Könenkamp, R. Appl. Phys. Lett. 2011, 99, 041106.

(17) Schenk, M.; Krüger, M.; Hommelhoff, P. Phys. Rev. Lett. 2010, 105, 257601.

(18) Kim, S.; Jin, J.; Kim, Y.-J.; Park, I.-Y.; Kim, Y.; Kim, S.-W. Nature 2008, 453, 757-760; See also comment in Nature 2012, 485, E1-E3.

(19) Zherebtsov, S.; et al. Nat. Phys. 2011, 7, 656-662.

(20) Zuloaga, J.; Nordlander, P. Nano Lett. 2011, 11, 1280.

(21) Hohenester, U.; Trügler, A. Comput. Phys. Commun. 2012, 183, 370-381.
(22) Garcia de Abajo, F. J.; Howie, A. Phys. Rev. B 2002, 65, 115418.

(23) Johnson, P. B.; Christy, R. W. Phys. Rev. B 1972, 6, 4370-4379.

(24) Corkum, P. B. Phys. Rev. Lett. 1993, 71, 1994-1997.

(25) Dombi, P.; Rácz, P. Opt. Express 2008, 16, 2887-2893.

(26) Yudin, G. L.; Ivanov, M. Y. Phys. Rev. A 2001, 64, 013409.

(27) Ropers, C.; Solli, D. R.; Schulz, C. P.; Lienau, C.; Elsaesser, T. Phys. Rev. Lett. 2007, 98, 043907.

(28) Keldysh, L. V. Sov. Phys. JETP 1965, 20, 1307-1314.

(29) Grubisic, A.; Ringe, E.; Cobley, C. M.; Xia, Y.; Marks, L. D.; Van Duyne, R. P.; Nesbitt, D. J. Nano Lett. 2012, 12, 4823.

(30) Trügler, A.; Tinguely, J. C.; Krenn, J. R.; Hohenau, A.; Hohenester, U. Phys. Rev. B 2011, 83, 081412(R).

(31) Krüger, M.; Schenk, M.; Hommelhoff, P.; Wachter, G.; Lemell, C.; Burgdörfer, J. New J. Phys. 2012, 14, 085019.

(32) Yalunin, S. V.; Gulde, M.; Ropers, C. Phys. Rev. B 2011, 84, 195426.

(33) Bormann, R.; Gulde, M.; Weismann, A.; Yalunin, S. V.; Ropers, C. Phys. Rev. Lett. 2010, 105, 147601. 